Document downloaded from:

http://hdl.handle.net/10251/126157

This paper must be cited as:

Torregrosa Cabanilles, C.; Molina Mateo, J.; Sabater I Serra, R.; Meseguer Dueñas, JM.; Gómez Ribelles, JL. (2018). Fluctuations of conformational mobility of macromolecules around the glass transition. Physical Review E. 97(6):062605-1-062605-7. https://doi.org/10.1103/PhysRevE.97.062605

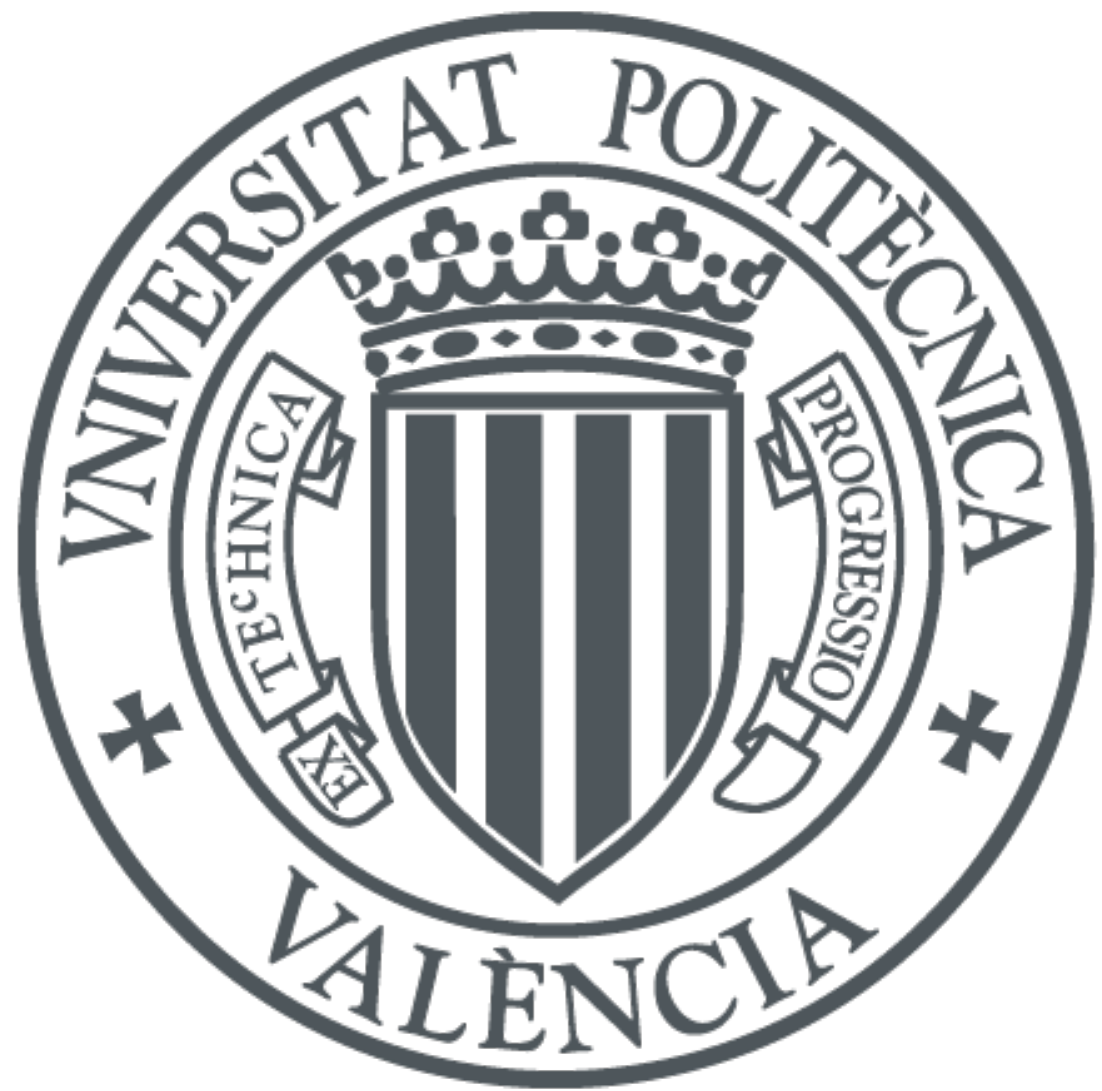

The final publication is available at

http://doi.org/10.1103/PhysRevE.97.062605

Copyright American Physical Society

Additional Information 


\title{
Fluctuations of conformational mobility of macromolecules around the glass transition
}

\author{
C. Torregrosa Cabanilles, ${ }^{*}$ J. Molina-Mateo, R. Sabater i \\ Serra ${ }^{\dagger}$ J.M. Meseguer-Dueñas, ${ }^{\dagger}$ and J.L. Gómez Ribelles ${ }^{\dagger}$ \\ Center for Biomaterials and Tissue Engineering, \\ Universitat Politècnica de València, Spain
}

(Dated: May 28, 2018)

\begin{abstract}
The heterogeneity of local dynamics in disordered systems is behind some key features of glass transition. In order to improve our understanding of the molecular dynamics in disordered systems in the vicinity of the glass transition, different parameters have been proposed to quantitatively describe dynamical heterogeneity. In the case of polymers, free volume models relate the macromolecular mobility to the free or accessible volume. The relationship between dynamic heterogeneity and fluctuations of accessible volume seems straightforward. In the present work, the heterogeneity of local dynamics in polymeric systems is analyzed by computer simulation with the Bond Fluctuation Model. The value of the accessible volume around each polymer chain is evaluated from a snapshot or static structure at each system state, resulting in a distribution of accessible volume that reflects system heterogeneity. The relationship between the average value and the standard deviation of free volume distributions at different temperatures fits a master curve for different systems, regardless of the specific inter- and intra-molecular interaction potentials that define each material. The dynamic slowdown around the glass transition is accompanied by a clear evolution of the mean value and shape of the accessible free volume distribution. The relative fluctuation of the dynamically accessible volume has been used as a parameter to quantitatively describe heterogeneity. The fluctuation varies with temperature with remarkable differences between the liquid and glassy states of the systems studied, presenting a peak at the glass transition temperature, which can be interpreted as a reflection of the distribution of local glass transition temperatures.
\end{abstract}

\footnotetext{
* ctorregr@fis.upv.es

$\dagger$ Also at CIBER-BBN, Biomedical Research Networking Center in Bioengineering, Biomaterials and Nanomedicine, Spain
} 


\section{INTRODUCTION}

The relationship between spatial distribution of molecules or polymer chain segments and their mobility in disordered liquids is still an open question in Physics. The mobility of individual entities in the disordered liquid becomes extremely dependent on their neighborhood, as density increases with decreasing temperature. Near the glass transition, a jump of a segment to a different position in the space requires the simultaneous or previous motion of a number of close molecular groups in a cooperative rearrangement that involves a region called the cooperative rearranging region, CRR [1]. The characteristic length of a CRR around the glass transition is in the order of 1 to $3 \mathrm{~nm}$ [2-4]. On cooling from the liquid state, molecular packing can vary from one point to the other, yielding at a given temperature to regions with significant fluctuations in density around the mean value. Interestingly, it has been shown that in the vicinity of the glass transition very small differences in local density can produce differences in the rearrangement rate or in the relaxation time of up to three or four orders of magnitude (see perspective in [5]). On the molecular scale, each small region of the amorphous material is surrounded by other similar regions whose disposition decisively influences the mobility of the first. The fluctuations in the conformation of each region and its neighbors result in non-homogeneous local dynamics. This phenomenon has been called dynamic heterogeneity [6-10] and originates in the variety of different local configurations that occur in disordered structures. Dynamic heterogeneity describes the spatial and temporal fluctuations in local dynamical behavior producing, for example, non-exponential relaxations in the evolution of the physical variables of the material. Experimental results on a microscopic scale support the presence of spatial and temporal fluctuations of the local dynamics of the glassy material [11]. The relationship between dynamic heterogeneity and fluctuations of free volume thus seems straightforward [12-14]. It has been suggested that an instantaneous picture of the structure of the material, especially a picture of the distribution of free volume fluctuations, could predict its dynamics and a number of computer simulation studies have been carried out on different disordered dense liquids with this aim [12-15]. Numerical Monte Carlo simulations can monitor different local variables such as relaxation times [16], the dynamically accessible volume [17] (DAV) and a recent similar concept, the probability of segment movement [18] (PSM).

The concept of the available volume of an atom was introduced some decades ago to 
explain certain qualitative properties of simple liquids [19]. Free volume models (see perspective in [20]) were also used to explain the temperature-dependence of the dynamic and mechanical properties of glass-forming systems, including polymers. The dynamically accessible volume (DAV) was introduced to be used in computer simulations of polymeric systems [17]. Following this line, some of the present authors proposed a DAV formulation for thermal systems to measure dynamic heterogeneity in Monte Carlo simulations of polymeric materials [21]. The importance of free volume in controlling glass transition phenomena has recently been pointed out [20]. Monte Carlo simulations of DAV distributions show the dynamic heterogeneity that occurs around the glass transition in polymers [22]. The DAV distributions represent the mobility of the chains of a polymeric material in the short term of a Monte Carlo step (MCS). These distributions show how the simulated macromolecules in a disordered state have different mobilities, depend on temperature and evolve with structural relaxation. Different parameters arise from these distributions describing the state, often non-equilibrium, related to the glass transition temperature and the structural relaxation process of the material. Although the distribution of relaxation times has also been used for the same purposes [23] as DAV, long simulation times are required to obtain them. This is suitable for systems in equilibrium but rules it out for non-equilibrium systems that evolve over time as in the case of structural relaxation processes. On the other hand, the distributions of other parameters like DAV and PSM [18] can be obtained from a snapshot of the material during the Monte Carlo simulation. Both variables are related to the mobility of the material in the short term of one MCS $[17,18]$. There is a connection between the DAV and PSM distributions and the equilibrium or non-equilibrium state of the material [22]. Different DAV distributions obtained from simulations of equilibrium and non-equilibrium states around the glass transition and during structural relaxation showed the relationship between DAV fluctuations and the different states of the system [22].

The aim of this work was thus to study the DAV distributions obtained from Monte Carlo simulations, choosing the appropriate distribution parameters to distinguish between the states of liquid in equilibrium and those of a non-equilibrium glass from a snapshot of the material structure. The DAV fluctuation, determined by the system snapshot (the chain segment positions at a given time during the simulation) together with information on the interaction potentials and temperature, as a measure of dynamic heterogeneity, is found to identify the state of the system around the glass transition. 


\section{SIMULATIONS}

\section{A. Model Construction}

The Bond Fluctuation Model is a coarse-grained Monte Carlo model frequently used to simulate the behavior of polymeric materials and reproduces their main features [16, 24]. It consists of a cubic lattice in which molecular groups occupy the empty space forming cubes. These groups can be bounded to form the chains of the polymeric material. The dynamics of the model consists of randomly choosing a molecular group and a direction of movement. The movement is performed with a probability given by the Metropolis criterion [25] as

$$
P=\min \left[1, \exp \left(-\frac{\Delta E}{k T}\right)\right]
$$

which takes into account the energy variation $\Delta E$ caused by the evaluated movement, the temperature $T$, and the Boltzmann's constant $k$. In this study, three interaction potentials were chosen to govern the system [16]. The first was the bond length intramolecular potential defined as

$$
U(l)=U_{0}\left(l-l_{0}\right)^{2}
$$

where the minimal energy distance is $l_{0}=3$ lattice units. The second was the bond angle intramolecular potential

$$
V(\theta)=V_{0}\left(\cos \theta-\cos \theta_{0}\right)^{2}
$$

with a minimal energy angle $\theta_{0}$ equal to $\pi$ radians. The third one was the Lennard-Jones intermolecular potential applied to all non-bonded groups up to a distance of four lattice units:

$$
U_{L J}=4 \varepsilon\left[\left(\frac{\sigma}{r}\right)^{12}-\left(\frac{\sigma}{r}\right)^{6}\right]
$$

with $\sigma=2$ lattice units.

The parameters $U_{0}, V_{0}$ and $\varepsilon$ control the interaction intensities of each of the potentials: bond length, bond angle and Lennard-Jones respectively. Their selected values are shown in Table I, representing three different material behaviors. These three different systems are introduced in order to be able to obtain general conclusions that do not depend on a specific map of energies. System 1 includes both the bond length and Lennard-Jones potentials of flexible chains. Higher intensities of these potentials, together with a bond angle potential, define System 2, resulting in considerably stiffer chains. And System 3 is characterized by 
TABLE I. Values of the interaction potential intensity parameters of the three systems: System 1 corresponding to flexible chains, System 2 includes higher potential intensities and a bond angle potential resulting in stiffer chains and System 3 is characterized by longitudinal flexibility but high torsional stiffness. The last column shows the glass transition temperature found for each system, calculated as usual from the energy evolution curve during the slow cooling ramp.

\begin{tabular}{ccccc}
\hline \hline System & $U_{0}$ & $V_{0}$ & $\varepsilon$ & $k T_{g}$ \\
\hline 1 & 0.75 & 0.0 & 0.25 & 0.2 \\
2 & 1.00 & 1.0 & 1.00 & 1.0 \\
3 & 0.10 & 1.0 & 1.00 & 0.9 \\
\hline \hline
\end{tabular}

a lower bond length potential intensity which results in a lower longitudinal stiffness that could facilitate crystallization.

Some variables were calculated during the simulations: the average energy per group as well as the bond length, bond angle and Lennard-Jones energy. The squared radius of gyration [16] was calculated as

$$
\left\langle R_{g}^{2}(N)\right\rangle=\frac{1}{N} \sum_{i=1}^{N}\left\langle\left(\boldsymbol{r}_{i}-\boldsymbol{r}_{c m}\right)^{2}\right\rangle
$$

where $N$ is the number of monomeric groups of the chains, $\boldsymbol{r}_{c m}$ is the position of the center of mass of the chain and $\boldsymbol{r}_{i}$ is the position of every group $i$ of the chain.

Simulations were performed in a cubic box with side $L=40$ lattice units, with periodic boundary conditions and density 0.5 . All systems were formed by 400 polymeric chains with 10 molecular groups each. Previous simulations with the parameters of System 1 showed a glass transition in cooling ramps and structural relaxation in isothermal annealing below the glass transition interval [22]. The thermal history began with an initial equilibration period of $10^{5}$ Monte Carlo steps (MCS) at temperature $k T=5$, which led to a liquid in equilibrium in all three systems. This initial period was followed by a cooling ramp at a constant rate of 0.1 units of $k T$ per every $10^{3}$ MCS until $k T=0.01$ (fast cooling ramp) or 0.1 units of $k T$ per every $2 \cdot 10^{4}$ MCS until $k T=0.05$ (slow cooling ramp). Additionally every system was simulated with specific isothermal annealing periods at temperatures around their glass transition interval for $10^{7} \mathrm{MCS}$, from the states attained at the cooling ramp. 


\section{B. Dynamically Accessible Volume}

The available volume of an atom at a given temperature $T$ was defined as a measure of the volume in which the motion of the atom takes place [19]:

$$
v=\int \exp \left(-\frac{U(r)}{k T}\right) d^{3} r
$$

where $U(r)$ was the potential energy of the atom, $k$ is Boltzmann's constant and $T$ is the temperature of the system. The dynamically accessible volume (DAV) for thermal systems [21] that we propose as a measure of the dynamic heterogeneity is simply an adaptation to the lattice Monte Carlo simulation model of the available volume. The DAV takes into account the probability of every empty cell being occupied in one MCS. The average value of this probability in the simulation box provides a measure of the free volume fraction that contributes to the mobility of the molecules:

$$
\langle D A V\rangle=\frac{1}{L^{3}} \sum_{j=1}^{L^{3}}\left\{\min \left[1, \sum_{i=1}^{n} \exp \left(-\frac{\Delta E_{i, j}}{k T}\right)\right]\right\},
$$

where $L$ is the box simulation size in lattice units, $n$ is the number of neighbors around a cell and $\Delta E_{i, j}$ is the variation in the energy caused by the movement of a group from cell $i$ to $j$. A DAV value is assigned to each polymer chain [22] as the average value of the DAV for each empty accessible cell that can be occupied in one MCS by a segment or group of that chain:

$$
D A V=\frac{1}{m} \sum_{j=1}^{m}\left\{\min \left[1, \sum_{i=1}^{n} \exp \left(-\frac{\Delta E_{i, j}}{k T}\right)\right]\right\},
$$

where $m$ is the number of empty accessible cells of a polymer chain, so that its DAV is related to the mobility of the specific polymer chain. The DAV distribution is a consequence of the different neighborhoods of each chain, it can be calculated at every step during the simulation and evolves with the state of the system. The distribution of the chain's DAV of a system state provides a physical picture of the dynamic heterogeneity of that state.

\section{RESULTS}

Fig. 1 shows the evolution of the average molecular group energy during the slow cooling ramp, with an equilibrium liquid line at high temperatures and decreasing energy as temperature decreases until reaching the glass transition temperature for each system (Table I). 


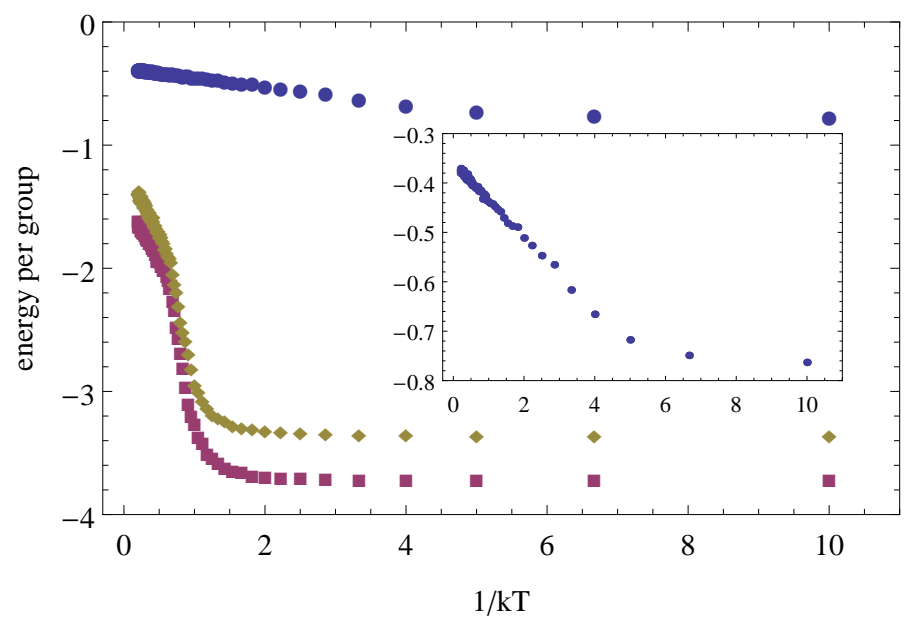

FIG. 1. Energy per molecular group according to the reciprocal of temperature during the slow cooling ramp for Systems 1 (circles), 2 (rhombus) and 3 (squares). The inset shows a zoom of the System 1.

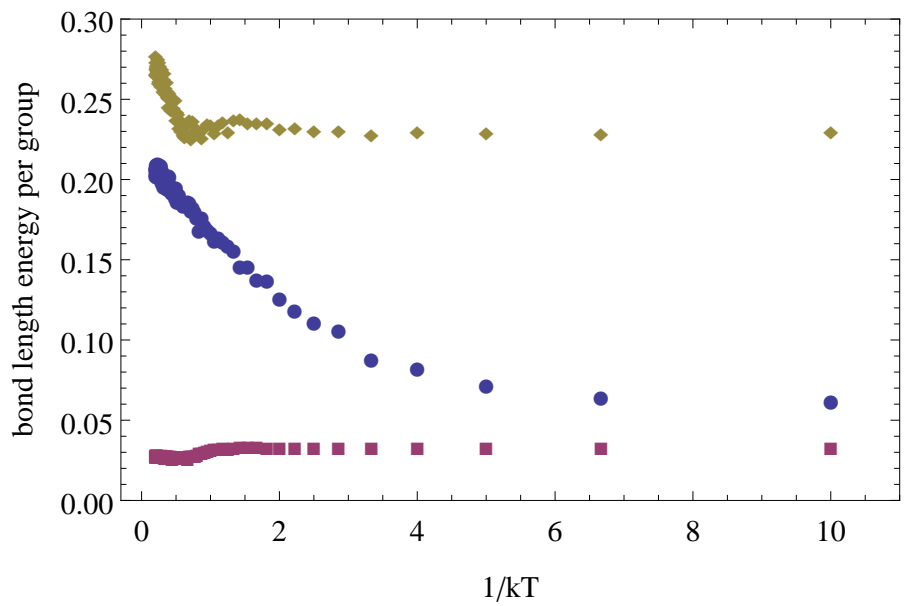

FIG. 2. Bond length potential energy per molecular group according to the reciprocal of temperature during the slow cooling ramp for Systems 1 (circles), 2 (rhombus) and 3 (squares).

Fig. 2 and Fig. 3 show, respectively, the bond length potential energy per group (Eq. 2) and the averaged radius of gyration of the chains (Eq. 5) during the slow cooling ramp to show the different behavior of the three systems. The evolution of both magnitudes stops below their glass transition temperatures, reaching different but constant values at each system.

Three examples of the DAV distributions of the chains obtained at each state during the cooling ramp of every system are shown in Fig. 4. The highest DAV values were obtained at 


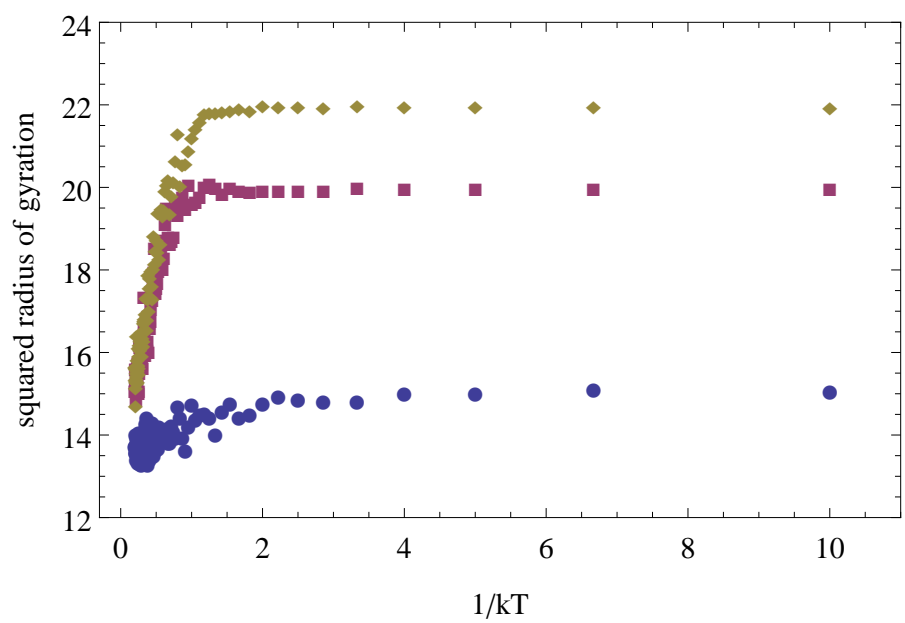

FIG. 3. Average molecular radius of gyration according to the reciprocal of temperature during the slow cooling ramp for Systems 1 (circles), 2 (rhombus) and 3 (squares).

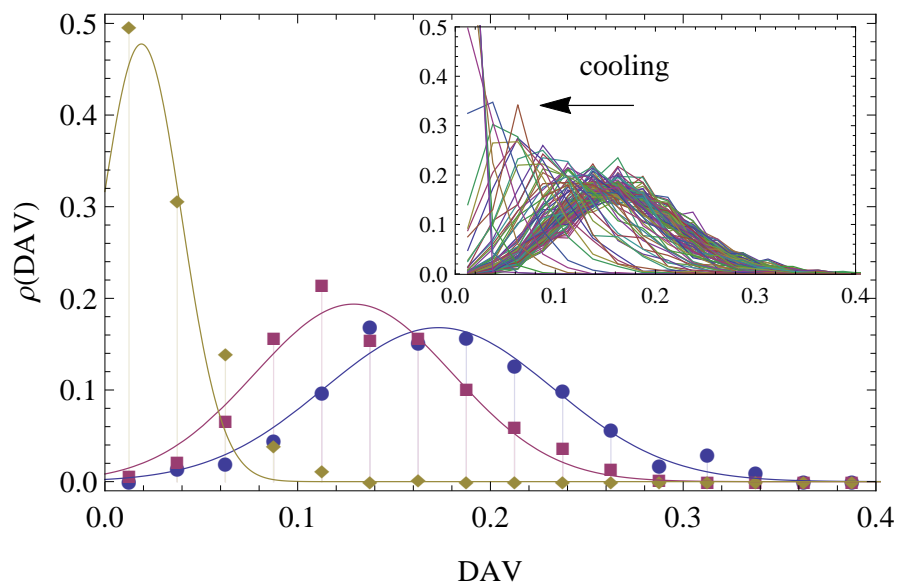

FIG. 4. DAV distributions (dots) of three System 1 states at temperatures $k T=5.0$ (circles), $k T=1.3$ (squares) and $k T=0.3$ (rhombus) during the slow cooling ramp. Lines represent the normal curve fitting for each distribution. The inset shows all DAV distributions obtained during the slow cooling ramp of System 1.

the highest temperatures. As the temperature drops, the distributions of the three systems are displaced to small DAV values and become narrower (only System 1 is shown).

In order to characterize the DAV distribution of each state and its evolution with temperature, several statistical distributions were tested for fit. The parameters that best characterized the chains' DAV distributions were found by fitting normal distributions (see Fig. 4). The fit of the DAV's distribution of each state on the cooling ramp gave the mean 


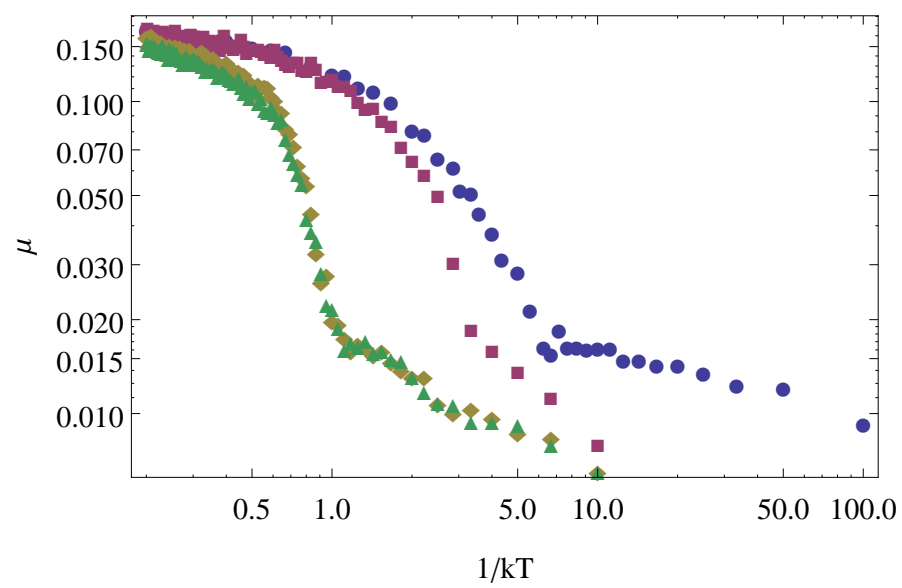

FIG. 5. Mean DAV value $\mu$ of states at different temperatures during the slow cooling ramp of System 1 (squares), 2 (triangles) and 3 (rhombus) and during the fast cooling ramp of System 1 (circles) according to the reciprocal of temperature.

DAV value $\mu$ and the width $\sigma$ for each state. This fitting was simply used as a tool to model all distributions with a small number of parameters.

The graph of the DAV mean value $\mu$ versus the reciprocal of temperature (Fig. 5) shows the equilibrium curve followed by the typical change in the slope related to the glass transition during the slow cooling ramp in the three systems. The results of the fast cooling ramp are only given for System 1 and show the displacement towards higher values of accessible volume with increasing cooling rate, as expected. This means that the evolution of the average value for the available free space for every molecule has a different behavior that depends on the state of the system.

The width $\sigma$ of the DAV distributions also decreases with temperature during the cooling ramp. Figure 6 shows a greater variation of $\sigma$ around the glass transition region for each system.

In order to study the evolution of these distributions we represented $\sigma$ versus $\mu$ for all three systems (Fig. 7). The difference between the liquid and the glassy behavior can again be clearly seen. While temperature is dropping during the cooling ramp, a linear relationship between the average value and the standard deviation of distributions of high temperatures is clearly visible. It should be noted that all three systems show this relationship regardless of their specific temperature. All points of the three systems fit into a master curve that clearly 


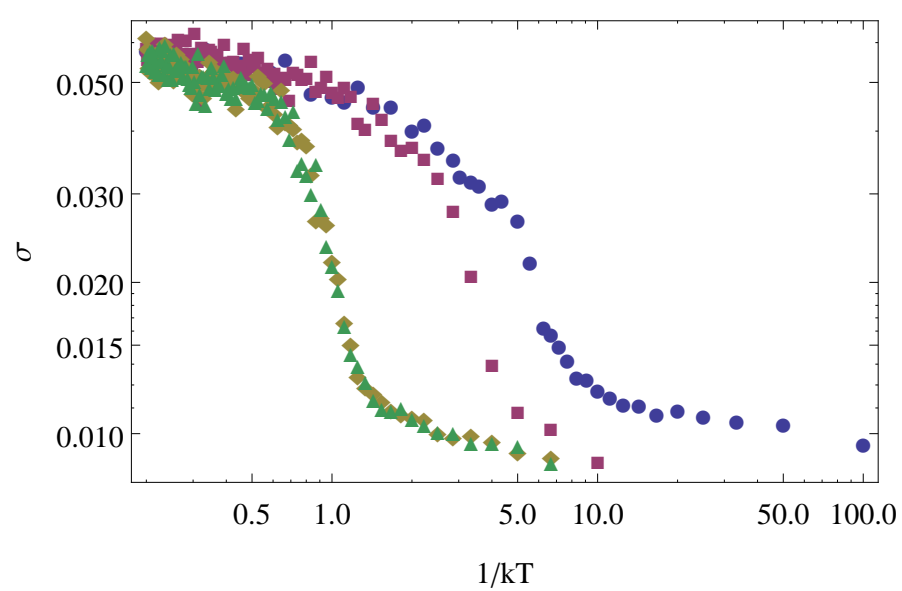

FIG. 6. Width value $\sigma$ of the distributions for states at different temperatures during the slow cooling ramp of System 1 (squares), 2 (triangles) and 3 (rhombus) and during the fast cooling ramp of system 1 (circles) according to the reciprocal of temperature.

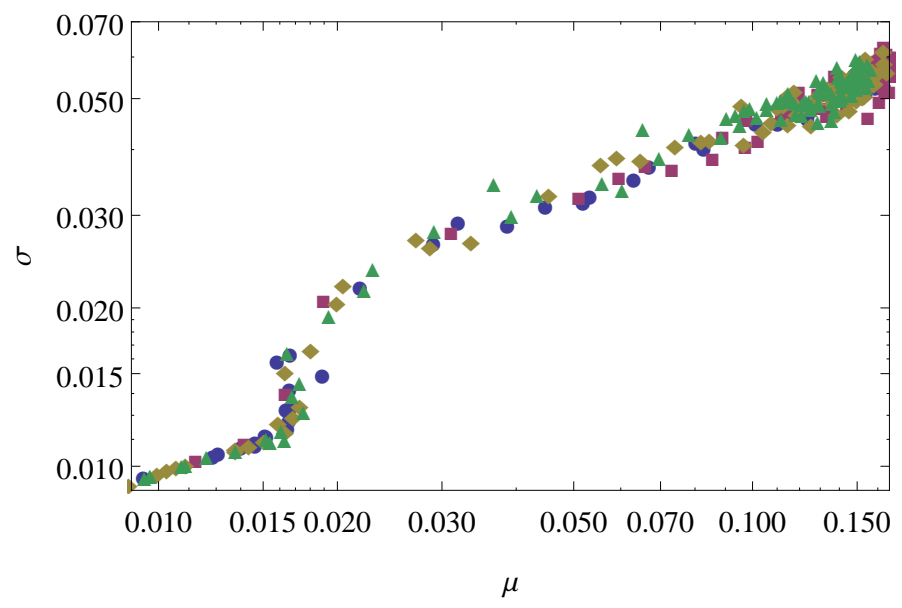

FIG. 7. Width $\sigma$ of the normal distributions against the mean DAV value $\mu$ of states at different temperatures during the slow cooling ramp of System 1 (squares), 2 (triangles) and 3 (rhombus) and during the fast cooling ramp of System 1 (circles).

establishes a strong correlation between the average value and the width of the distribution in the liquid state. Furthermore, the different glass transition temperatures can be seen in this graph as a loss of this relationship when $\sigma$ falls steeply for a given value of $\mu$, which is the same for all the systems.

This linear relationship between $\sigma$ and $\mu$ is broken as a step around the value $\mu=0.017$ 


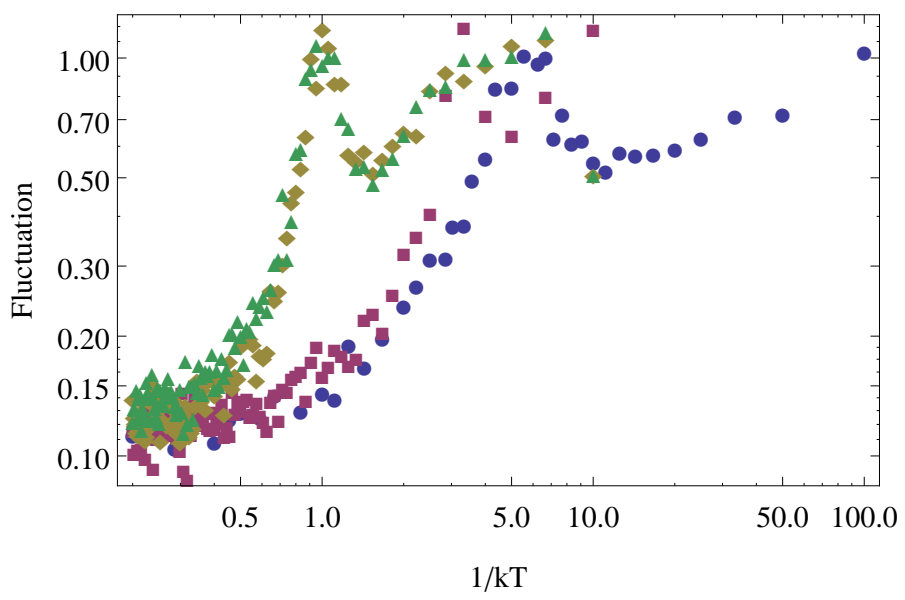

FIG. 8. Relative fluctuation $\delta$ according to the reciprocal of temperature during the slow cooling ramp of System 1 (squares), 2 (triangles) and 3 (rhombus) and during the fast cooling ramp of System 1 (circles).

and is the same for the three different systems. This mean DAV value at the step is for different temperatures in each system around their glass transition temperature, as can be seen in Fig. 5. The width $\sigma$ of the distributions changes faster around the glass transition region of each system. After this transition there is a new linear relationship for the glassy state in all systems.

As a result of the DAV fluctuations throughout each system, the width of the DAV distribution is related to the dynamic heterogeneity of the system state. The relative fluctuation of DAV can thus be chosen as a measure of the dynamic heterogeneity of the system. Figure 8 shows the relative fluctuation of DAV

$$
\delta=\left\langle\left(\frac{D A V-\mu}{\mu}\right)^{2}\right\rangle=(\sigma / \mu)^{2}
$$

against temperature. The relative fluctuation $\delta$ gets higher as the temperature drops, with values between 0.5 and 1 at temperatures below the glass transition of each system. Liquid states above the glass transition temperature show values of the relative fluctuation below 0.5. The three systems also show a peak of the relative DAV fluctuation around the glass transition temperature, where the relative fluctuation reaches values around one.

Figure 9 shows the relationship found between fluctuation and $\mu$, which is the same for the three different systems. The fluctuation value at the step is for different temperatures 


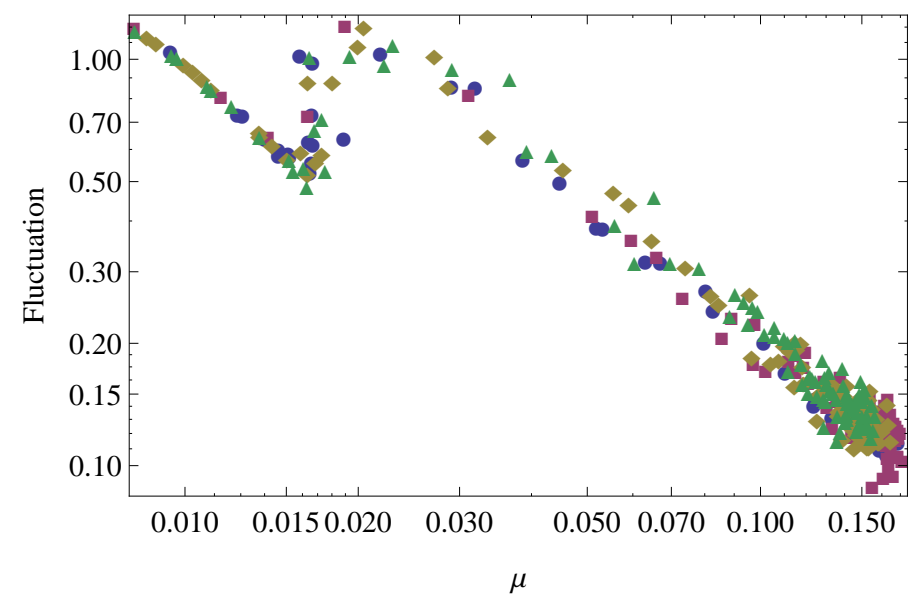

FIG. 9. Relative fluctuation $\delta$ as a function of the mean DAV value $\mu$ during the slow cooling ramp of System 1 (squares), 2 (triangles) and 3 (rhombus) and during the fast cooling ramp of System 1 (circles).

in each system and are in the region of their glass transition temperature.

\section{DISCUSSION}

DAV computes the fraction of lattice vacancies that could be occupied by a jump of a neighboring chain segment, according to the probability of this happening, which takes into account the energy change involved and temperature through the Metropolis criterion. In this work, we calculate the accessible volume per chain, i.e. the fraction of vacancies that can be occupied around the segments of a polymer chain. In this way, DAV per chain is not merely a measure of the local free space around a polymer segment but a measure of the segment mobility in a given conformation of the whole system. This formulation determines local fluctuations of accessible free volume around the mean value and so also characterizes dynamic heterogeneity. The distributions of accessible volume were calculated in simulations of disordered polymer materials with the Bond Fluctuation Model. Three cases were studied with different intra and intermolecular interaction potentials: the first with flexible chains and weak intermolecular interactions, the second with stiffer chains and stronger intermolecular interactions and the third allowing more flexibility of the chain's longitudinal direction.

The DAV distributions were obtained in both equilibrium and non-equilibrium states 
and compared in different conditions of the model materials. This analysis does not require any data on the time evolution or system dynamics, unlike the relaxation times distribution analysis used in previous studies [23], which cannot study out-of-equilibrium states. It is important to note that the distribution of accessible volume is obtained purely from the static structure of the system, i.e. the position of the groups of the polymer chains (a "snapshot"), together with information on the interaction potentials and temperature. Due to the absence of "universal" parameters that characterize the temperature-dependent heterogeneity data [10], the distribution of accessible volume is studied to quantitatively describe heterogeneity. Unlike other heterogeneity parameters, it is calculated from only a single "snapshot" or static structure of the system. The DAV distributions describes dynamic heterogeneity in simulations of polymeric materials [22] and show how distinct regions or chains of the system in a disordered state have different values of accessible volume and therefore distinct mobility in the short term of 1 MCS.

The DAV distributions are found to fit well with normal distributions and therefore can be mathematically characterized by their mean value $\mu$ and width $\sigma$. The mean DAV value presents a transition of around the same value $\mu=0.017$ and temperatures around the $k T_{g}$ characteristic of each system, the glass transition temperature calculated by the usual methods from the energy curves versus temperature in a cooling ramp. The width of the distributions, $\sigma$, increases with temperature and changes abruptly around the glass transition temperature of each system. The relative spatial fluctuation of the DAV, $\delta=(\sigma / \mu)^{2}$, is proposed as a measure of the dynamic heterogeneity in the system and increases by an order of magnitude from the liquid state, at high temperatures, to the glassy state below the glass transition. At high temperatures, the high segmental mobility yields more homogeneous local configurations, with a DAV fluctuation value between 0.1 and 0.15 . However, on cooling, regions of the disordered material with sizes of the order of the CRR, frozen in conformations with more or less free volume and consequently larger fluctuations of accessible free volume appear in the glassy state.

Although the mobility of macromolecules is a dynamic property, it is related to static properties as the local density or the local conformation. Small changes of these static properties produce very important differences of the mobility. The spatial fluctuations in the conformation of each region have been measured around the glass transition temperature through the dynamically accessible volume. The DAV is a static property, calculated from 
a snapshot of the simulated system and the knowledge of the interaction potentials. The fluctuation of DAV shows important differences between the liquid and glassy states of the studied systems. The relative fluctuation increases an order of magnitude from the liquid to the glassy states and shows a maximum around the glass transition temperature.

The order of magnitude of dynamic heterogeneity, both in the liquid and the glassy state, seems to be determined by the average DAV (see Fig. 9). It should be noted that a simple static analysis of a given configuration can be enough to distinguish between the liquid and the glassy state with no additional simulations. The distribution of dynamic heterogeneity is proposed as a tool to characterize the chain mobility of a system at temperatures close to the glass transition temperature. The fluctuations calculated from a snapshot of the material during the simulation allow to identify and characterize the state and dynamics of the system. Interestingly enough, a peak appears in the dynamic heterogeneity in the middle of the glass transition in all the model materials. This peak must be interpreted as a consequence of the presence of a distribution of glass transition temperatures. Dynamic heterogeneity itself makes that, at the start of the glass transition, local mobility freeze at higher temperatures in rearrangement regions with more packed conformations, while others with more DAV still behave as in the liquid state.

When the temperature drops further, the number of frozen CRR increases and DAV fluctuation is reduced, i.e. the system becomes more homogeneous. At intermediate temperature within the range in which the macroscopic glass transition takes place, the DAV fluctuation goes through a maximum. In this way, dynamic heterogeneity is responsible for the width of the temperature interval of the glass transition, which in amorphous polymers has been shown to be dependent on chain stiffness and other parameters such as the distribution of molecular weight, or the presence of side groups along the chain. The Monte Carlo simulations also show that the influence of these molecular parameters on dynamic heterogeneity can be mediated by the average DAV value.

\section{CONCLUSIONS}

As the structure and interaction potentials determine the distribution of accessible volume of a given state of the simulated BFM system, DAV average and relative fluctuation values can be assigned to each system state. DAV distributions are a measurement of the 
mobility of the system that can be performed in simulations on both equilibrium and nonequilibrium states. The relative DAV fluctuation determines the dynamic characteristics of the state, differentiating the liquid from the glassy states and identifying the glass transition temperature region. The results of the simulations of three systems of different potentials indicate that high values of the relative fluctuation belong to glassy states, with a peak at the glass transition region, which reveals the distribution of local glass transition temperatures. The low values belong to liquid states. These results show the relationship between structure, DAV distribution and dynamics, addressing the problem of quantifying dynamic heterogeneity using purely structural information. The universality of these relationships with other lattice and molecular dynamics models will be tested in future studies.

\section{ACKNOWLEDGMENTS}

The support from the Ministry of Economy and Competitiveness (Spain) through the project MAT2016-76039-C4-1-R (including the FEDER financial support) is gratefully acknowledged. CIBER-BBN is an initiative funded by the VI National R\&D\&I Plan 2008-2011, Iniciativa Ingenio 2010, Consolider Program. CIBER Actions are financed by the Instituto de Salud Carlos III with assistance from the European Regional Development Fund.

[1] G. Adam and J. H. Gibbs, J. Chem. Phys. 43, 139 (1965).

[2] E. Donth, J. Non-Cryst. Solids 53, 325 (1982).

[3] E. Donth, J. Non-Cryst. Solids 131-133, 204 (1991).

[4] J. L. Gómez Ribelles, A. Vidaurre Garayo, J. M. G. Cowie, R. Ferguson, S. Harris, and I. J. McEwen, Polymer 40, 183 (1999).

[5] G. B. McKenna and S. L. Simon, Macromolecules 50, 6333 (2017).

[6] G. Diezemann, J. Chem. Phys. 107, 10112 (1997).

[7] M. D. Ediger, Annu. Rev. Phys. Chem. 51, 99 (2000).

[8] J. P. Garrahan and D. Chandler, Phys. Rev. Lett. 89, 035704 (2002).

[9] L. Berthier, Physics 4, 42 (2011).

[10] X. Di and G. B. McKenna, J. Chem. Phys. 138, 12A530 (2013). 
[11] D. Bingemann, R. M. Allen, and S. W. Olesen, J. Chem. Phys. 134, 024513 (2011).

[12] H. Shiba and T. Kawasaki, J. Chem. Phys. 139, 184502 (2013).

[13] N. B. Tito, J. E. G. Lipson, and S. T. Milner, Soft Matter 9, 3173 (2013).

[14] X. Yang, R. Liu, M. Yang, W. H. Wang, and K. Chen, Phys. Rev. Lett. 116, 238003 (2016).

[15] S. P. Pan, S. D. Feng, J. W. Qiao, W. M. Wang, and J. Y. Qin, J. Alloys Compd. 664, 65 (2016).

[16] K. Binder, Monte Carlo and Molecular Dynamics Simulations in Polymer Science (Oxford University Press, 1995).

[17] K. A. Dawson, A. Lawlor, P. De Gregorio, G. D. Mccullagh, E. Zaccarelli, and P. Tartaglia, Physica A 316, 115 (2002).

[18] Y. Nie, X. Ye, Z. Zhou, W. Yang, and L. Tao, J. Chem. Phys. 141, 074901 (2014).

[19] H. J. Bernstein and V. F. Weisskopf, Am. J. Phys. 55, 974 (1987).

[20] R. P. White and J. E. Lipson, Macromolecules 49, 3987 (2016).

[21] J. Molina-Mateo, J. M. Meseguer-Dueñas, and J. L. Gómez-Ribelles, Macromol. Theory Simul. 15, 32 (2006).

[22] J. Molina-Mateo, C. Torregrosa-Cabanilles, R. Sabater-Serra, J. M. Meseguer-Dueñas, and J. L. Gómez-Ribelles, J. Non-Cryst. Solids 362, 175 (2013).

[23] J. Molina-Mateo, J. Meseguer Dueñas, J. Gómez Ribelles, and C. Torregrosa Cabanilles, Polymer 50, 5618 (2009).

[24] I. Carmesin and K. Kremer, Macromolecules 21, 2819 (1988).

[25] N. Metropolis, A. W. Rosenbluth, M. N. Rosenbluth, A. H. Teller, and E. Teller, J. Chem. Phys. 21, 1087 (1953). 